Chronic Obstructive Pulmonary Diseases: Journal of the COPD Foundation

\title{
Editorial
}

\section{Patent Foramen Ovale in COPD and Hypoxia: Innocent Bystander or Novel Therapeutic Target?}

\author{
Brett E. Fenster, MD, ${ }^{1}$ John D. Carroll, MD $^{2}$
}

\begin{abstract}
Abbreviations: patent foramen ovale, PFO; transcranial Doppler ultrasonography, TCD; Global initiative for chronic Obstructive Lung Disease, GOLD; partial pressure of oxygen, $\mathbf{P}_{\mathrm{a}} \mathbf{O}_{2}$; oxygen uptake, $\mathbf{V}^{\prime} \mathbf{O}_{2}$; high-intensity transient signals, HITS

Funding Support: Not Applicable.

Citation: Fenster BE, Carroll JD. Patent foramen ovale in COPD and hypoxia: Innocent bystander or novel therapeutic target? J COPD F. 2014;1(2):151-154. doi:http:dx.doi.org/10.15326/jcopdf.1.2.2014.0146
\end{abstract}

\section{Address correspondence to:}

Brett E. Fenster, MD

Division of Cardiology

National Jewish Health

1400 Jackson Street, \#J328

Denver, CO 80206

Business Phone: (303) 398-1251

Fax: (303) 595-5282

fensterb@njhealth.org

\section{Keywords:}

COPD;patent foramen ovale;PFO;transcranial Doppler ultrasonography;TCD

\begin{tabular}{ll}
\hline 1 National Jewish Health, Division of Cardiology, Denver, \\
Colorado \\
\hline 2 University of Colorado-Denver, Division of Cardiology, \\
Aurora
\end{tabular}

The pathophysiologic origins of hypoxia in COPD involve multiple mechanisms, most notably ventilation perfusion mismatch as a consequence of airflow obstruction and emphysematous capillary bed destruction. However, there is increasing interest in understanding the role of patent foramen ovale (PFO)mediated right-to-left shunting in hypoxia as a potential therapeutic target. In this issue of Chronic Obstructive Pulmonary Diseases: Journal of the COPD Foundation, Martolini et al report on their findings regarding the functional significance of PFO in hypoxia and exercise limitation in moderately severe COPD. The authors performed transcranial Doppler ultrasonography (TCD) for identification of $\mathrm{PFO}$ during cardiopulmonary exercise testing in a small cohort of Global initiative for chronic Obstructive Lung Disease (GOLD) stage II individuals. A PFO was identified in 12 of 22 individuals, and $\mathrm{PFO}$ presence was itself an independent correlate of partial pressure of oxygen in arterial blood $\left(\mathrm{PaO}_{2}\right)$. Furthermore, PFO shunting appeared to increase with elevated respiratory muscle work and intrathoracic pressure swings as assessed by esophageal pressure manometry. However, and most importantly, PFO presence did not associate with markers of exercise performance including oxygen uptake $\left(\mathrm{V}^{\prime} \mathrm{O}_{2}\right.$ peak $\left./ \mathrm{kg}\right)$ or 6-minute walk distance. The authors conclude that $\mathrm{PFO}$ is unlikely to have a functional impact on exercise capacity in moderately severe COPD.

These findings are provocative and at first glance appear to dispel the notion that PFO shunting, either at rest or with activity, necessarily worsens exercise capacity through a hypoxia-dependent mechanism. On further examination, 2 key points emerge concerning the patients studied. First, the majority of PFO individuals had only mild/Grade I shunt, and the low threshold TCD criteria used may have included some with no PFO. Second, patients in both the PFO and nonPFO cohorts did not have significant resting hypoxia, or for that matter, exertional hypoxia. Thus the patients in the PFO group most likely had incidental PFO's, and it would not be expected that a functional impact on functional capacity would be found. Normoxic COPD populations with mild resting shunt are not the patients in whom the functional impact of a PFO will be manifest. Other studies have provided some insight but still 
fail to adequately address the clinical issue of whether management of hypoxic COPD patients with more substantial shunting present a unique therapeutic opportunity to improve function. Shaikh et al recently evaluated GOLD stage III and stage IV individuals with severe hypoxia $\left(\mathrm{PaO}_{2}<7.3 \mathrm{kPa}\right)$ and less severe hypoxia $\left(\mathrm{PaO}_{2}>8.0 \mathrm{kPa}\right)$. They found no significant difference in PFO prevalence by TCD examination (39\% vs. $52 \%$; $\mathrm{P}=0.27){ }^{1} \quad$ Furthermore, Kilic et al reported no significant difference in hypoxia in COPD individuals with and without PFO ( $46.5 \pm 13.77$ vs. 57.4 $\pm 6.85 \mathrm{mmHg}$, respectively), although only 4 individuals with a PFO were included in the study. ${ }^{2}$ The issue is not the prevalence of any PFO but the potential significant contribution to systemic hypoxemia of volumetrically substantial right to left shunting through an anatomically large PFO in some patients. The functional impact of that degree of shunting is difficult to dissect based on exercise testing. A more convincing and active approach to hypothesis testing is to eliminate the intracardiac shunting and then assess whether patients improve or not. Sham procedures might be necessary in a control group if subjective assessments of functional and quality of life changes are included in the study design.

An additional limitation of the present study is the use of TCD to define the presence of a PFO in a COPD cohort. TCD cannot discriminate between intracardiac shunting and intrapulmonary shunting. COPD patients can demonstrate significant intrapulmonary shunting that does not necessarily correlate with airflow, lung volume, or resting blood gas. ${ }^{3}$ Furthermore, large diameter $(>25-50 \mu \mathrm{M})$ intrapulmonary arteriovenous anastomoses are known to exist under physiologic conditions. ${ }^{4}$ Consistent with prior investigations using TCD in the cryptogenic stroke population, this study defined PFO presence by demonstrating single highintensity transient signals (HITS) within 25 seconds of saline contrast injection. ${ }^{5}$ However, intrapulmonary shunts can present with $<5$ HITS and therefore potentially masquerade as a $\mathrm{PFO}$ in this study. ${ }^{6}$ Because $66 \%$ of PFO patients had 1-10 HITS and the presence of PFO was not confirmed by echocardiography, the potential for concurrent intrapulmonary shunts to confound this study's results cannot be excluded.

This study adds to the growing body of work investigating the role of PFO in hypoxia and human performance. The role of PFO in contributing to hypoxia in diverse conditions such as obstructive sleep apnea, left ventricular assist device insertion, paralyzed hemidiapragm, orthodeoxia platypnea, and COPD require a careful approach separating incidental clinically-insignificant PFOs from those that are pathogenic. This study and others argue against a pathologic role for PFO in a significant part of the COPD patient spectrum. However, multiple case series have reported improvement in dyspnea and/or supplemental oxygen use following percutaneous closure in hypoxic populations including those with COPD. ${ }^{7,8}$

These conflicts in the literature may reflect publication biases but also important differences in patients studied and study designs. PFO closure studies are more likely to include patients with echocardiographically-detected large right-to-left intracardiac shunting. Second, PFO closure studies have been observational studies lacking a control group, randomization, and a standardized methodology of shunt detection, quantification, and assessment of change in functional state. While patient-reported outcomes are important, self-reported endpoints in unblinded participants can be confounded by patients' expectations. Another explanation involves the inclusion of heterogeneous patient cohorts in PFO closure studies that are defined by hypoxia as opposed to a specific pulmonary disease state. If PFOs in some patients do indeed impact systemic oxygenation and/ or exercise capacity via right-to-left shunting, one might anticipate that performing $\mathrm{PFO}$ closure in a population with severe/disproportionate hypoxia and dyspnea relative to background COPD may be more likely to demonstrate a benefit and support a pathologic role for PFOs.

Other methodological challenges have added to the complexity of this field. The contribution of rightto-left shunting via a PFO to systemic hypoxemia is difficult to quantify. Oxygen saturation of venous blood in all 4 pulmonary veins can be collected as well as the downstream systemic arterial blood after the PFO contribution has been mixed. However, there are uncertainties as to the volume of venous return in each vein, and there is no validated way to calculate the subsequent PFO contribution in clinical studies. Furthermore, studies performed in supine patients may have little relevance to the pathophysiology during exercise.

A well-planned study would also need to assess for concurrent pulmonary hypertension as a driver of PFO shunting. At what level of pulmonary hypertension or pulmonary vascular disease should PFO closure be 
contraindicated? The burden of proof may rest upon future percutaneous closure trials with randomization and perhaps patient and investigator blinding. Optimization and stabilization of other therapies for COPD must be achieved prior to randomization. There must be well-documented PFO anatomy and physiology at baseline and at 6-12 months time when closure is considered as complete as possible with detection and quantification of residual shunting that may occur in $10 \%-25 \%$ of patients. The study should be designed to objectively demonstrate improved oxygenation, functional capacity, and patient-reported outcomes. Finally, the implanted device as well as the implantation procedure must have an extremely low complication rate such that any potential benefit substantially counterbalances the possibility of harm. The time is right for such a multicenter study lead by a multidisciplinary group to more definitively determine if and when a PFO in COPD is an innocent bystander or a novel therapeutic target. 


\section{References}

1. Shaikh ZF, Kelly JL, Shrikrishna D, et al. Patent foramen ovale is not associated with hypoxemia in severe chronic obstructive pulmonary disease and does not impair exercise performance. Am J Respir Crit Care Med. 2014;189(5):540-547. doi: http:// dx.doi.org/10.1164/rccm.201309-16180C

2. Kilic H, Balci MM, Aksoy MN, et al. Patent foramen ovale among patients with mild chronic obstructive pulmonary disease and unexplained hypoxia. Echocardiography. 2010;27(6 ):687-690. doi: http://dx.doi.org/10.1111/j.1540-8175.2009.01105.x

3. Miller WC, Heard JG, Unger KM. Enlarged pulmonary arteriovenous vessels in COPD. Another possible mechanism of hypoxemia. Chest. 1984;86 (5):704-706. doi: http://dx.doi. org/10.1378/chest.86.5.704

4. Lovering AT, Stickland MK, Kelso AJ, Eldridge MW. Direct demonstration of 25- and 50-microm arteriovenous pathways in healthy human and baboon lungs. Am J Physiol Heart Circ Physiol. 2007;292 (4):H1777-81. doi: http://dx.doi.org/10.1152ajpheart. 01024.2006

5. Caputi L, Carriero MR, Falcone C, et al. Transcranial Doppler and transesophageal echocardiography: comparison of both techniques and prospective clinical relevance of transcranial Doppler in patent foramen ovale detection. J Stroke Cerebrovasc Dis. 2009;18(5):343-348. doi: http://dx.doi.org/10.1016/j.jstrokecerebrovasdis.2008.12.001

6. Nemec JJ, Marwick TH, Lorig RJ, et al. Comparison of transcranial Doppler ultrasound and transesophageal contrast echocardiography in the detection of interatrial right-to-left shunts. Am J Cardiol. 1991;68 (15):1498-1502. doi: http://dx.doi. org/10.1016/0002-9149(91)90285-S

7. Fenster BE, Nguyen BH, Buckner JK, Freeman AM, Carroll JD. Effectiveness of percutaneous closure of patent foramen ovale for hypoxemia. Am J Cardiol. 2013;112(8):1258-1262. doi: http:// dx.doi.org/10.1016/j.amjcard.2013.06.022

8. Ilkhanoff, L, Naidu S, Rohtagi S, Ross MJ, Silvestry FE, Hermann HC. Transcatheter device closure of interatrial septal defects in patients with hypoxia. J Interv Cardial. 2005;8 (4):227-232. doi: http://dx.doi.org/10.1111/j.1540-8183.2005.00043.x 